\title{
Ponctuation et connecteurs en français classique. Du reposoir (périodique) à la structure (phrastique)
}

\author{
Mathieu GoUX \\ Université de Caen Normandie \\ mathieu.goux@unicaen.fr \\ https://orcid.org/0000-0003-4211-8309
}

\section{Resumen}

En este artículo, analizamos la evolución de dos conectores aditivos (puis y ensuite) y de tres conectores adversativos del francés (cependant, néanmoins y pourtant) a lo largo del siglo XVII, en las ediciones príncipe de textos narrativos y argumentativos. Estudiamos la función que desempeñan estos conectores asociados a la puntuación y a la dinámica informativa de los enunciados. Demostramos que la función textual de tales conectores acompañó la transición de la escritura en periodos a la escritura de frases, transición que aportó a los autores nuevos recursos para organizar sus ideas. En particular, mostramos cómo estas nuevas perspectivas favorecieron el uso de cependant y reforzaron su papel argumentativo a lo largo del siglo XVII. Palabras clave: diacronía, conectores, período y oración, puntuación, cependant.

\section{Résumé}

Nous analysons l'évolution de deux connecteurs additifs (puis et ensuite) et trois connecteurs adversatifs (cependant, néanmoins, pourtant) au long du XVII ${ }^{\mathrm{e}}$ siècle dans les textes narratifs et argumentatifs. Nous étudions leur rôle en association de la ponctuation des éditions princeps et de la dynamique informationnelle des énoncés. Nous montrons que le rôle textuel des connecteurs a accompagné le passage de l'écriture périodique à l'écriture phrastique en donnant aux auteurs de nouvelles ressources d'organisation du propos. Nous montrons notamment comment cependant a bénéficié de ces nouvelles perspectives et a vu son rôle argumentatif renforcé au long du XVII ${ }^{e}$ siècle.

Mots-clés : diachronie, connecteurs, période et phrase, ponctuation, cependant.

\section{Abstract}

This paper analyze the evolution of two additive (puis and ensuite) and three adversarial (cependant, néanmoins, pourtant) connectors throughout the seventeenth century in narrative and argumentative texts. We study their role in association with the punctuation of the

*Artículo recibido el 15/09/2020, aceptado el 30/03/2021. 
editiones principes and the informational dynamics of the utterances. We show that the textual role of connectors accompanied the transition from periodical writing to phrasal writing, by giving authors new resources for organizing their discourses. In particular, we show how cependant benefited from these new perspectives and saw its argumentative roles reinforced throughout the seventeenth century.

Keywords: diachrony, connector, period and sentence, punctuation, cependant.

\section{Introduction}

La grammaire textuelle appliquée à la diachronie du français connaît, depuis quelques années, un intérêt certain de la part des chercheurs. Depuis les premières études de Bernard Combettes (1983), les travaux portant sur la fabrique de l'énoncé dans les états anciens de la langue se sont multipliés (Fournier, 1998 ; Goux 2019b; Le Goffic, 2011; Siouffi, 1997 ; Siouffi, 2020 ; Prévost, 2001 ; pour ne donner que quelques jalons), et ils ont exploré avec grand détail la façon dont la continuité référentielle (ou la cohésion textuelle) et la dynamique informationnelle (ou la cohérence textuelle) étaient effectivement gérées dans les énoncés du temps. Plus rares sont cependant les travaux se consacrant au troisième sujet d'étude privilégié de la grammaire de texte, la connexité textuelle, que nous définirons à la suite de Frank Neveu comme la notion renvoyant à certaines relations de cohésion dans un texte et plus largement, dans un discours, aux relations dont le fonctionnement linguistique et pragmatique est pris en charge par des marqueurs de connexité ou des connecteurs. Comme le précise Franck Neveu (2007 : 166), ces marqueurs opèrent principalement au niveau transphrastique et au niveau de la structure séquentielle du texte ou du discours, la connexité composant une propriété formelle fondamentale de ceux-ci.

On notera cependant que la définition proposée à l'instant est circulaire, puisque nous considérons que la connexité est prise en charge par les connecteurs... tandis que les connecteurs sont définis comme des marques de connexité. On peut certes considérer que la connexité est une notion englobant davantage que la seule classe des connecteurs puisqu'en elles-mêmes, les anaphores et la succession référentielle participent également à la connexité textuelle (Goux, 2020 ; Neveu, 2007), mais cela ne suffit cependant pas à arrêter la définition de ce concept. Il faudrait, ne serait-ce, que parvenir à définir ce que sont les connecteurs mais là encore, nous nous heurtons à un certain nombre de difficultés.

En effet, le terme générique de connecteur, que Riegel et al. définissent comme des « termes de liaison et de structuration " (2014 : 1044), est souvent défini a contrario, conséquence de leur grande variation morphologique, catégorielle et sémantique. Ce sont des expressions, mots ou groupes de mots, qui ne sont pas prédicatifs en euxmêmes, bien qu'ils servent à construire des prédications, et qui ne sont pas référentiels, bien qu'ils articulent la succession des référents entre eux. Ce sont généralement des 
adverbes ou des groupes adverbiaux (cependant, néanmoins...), mais l'on inclut également dans cette classe des conjonctions (mais, or) et certains syntagmes prépositionnels (d'abord, à première vue, d'une part...). Il faut noter cependant qu'à côté de cette définition générale, Neveu (2007), considère également comme des connecteurs les « constructions détachées", soit les participes apposés en position initiale de séquence, qu'analyse Combettes (1998); il s'agit cependant d'une position peu suivie par les chercheurs, et que nous ne considérerons pas dans notre contribution quand bien même ce type de constructions détachées, notamment en tête de séquence textuelle ou de chapitre, aurait d'évidents liens avec la connexité (Goux, 2020). À cette difficulté définitionnelle enfin, Franck Neveu toujours souligne avec précision la confusion qu'il peut exister entre les emplois du terme, sensiblement distincts selon l'approche que l'on choisit d'adopter (nous soulignons) :

[L]e terme de connecteur connaît trois principaux types d'emplois, étroitement corrélés. Le premier lui vient de la logique, où le terme peut être défini, par distinction avec le terme d'opérateur, comme un foncteur ayant pour argument une paire ordonnée de propositions. [...] Le deuxième emploi s'est développé en pragmatique, par les diverses approches non formalistes des connecteurs en langue naturelle [...] destinées à rendre compte des processus inférentiels déclenchés par l'usage de mots comme $e t$, mais, si. Le troisième emploi, plus englobant, s'est développé en sémantique discursive et textuelle pour désigner des mots ou des expressions (conjonctions, adverbes, syntagmes prépositionnels, etc.) dont la fonction est de lier des séquences discursives de nature phrastique ou textuelle à des fins de cohésion et de cohérence du discours (Neveu, 2007 : 165-166, les italiques sont nôtres).

Aussi, même lorsque nous sortons du domaine de la logique et de l'argumentation et que nous nous concentrons, comme nous le ferons ici, sur l'approche textuelle des connecteurs, la connexité est délicate à étudier puisque l'hétérogénéité catégorielle des connecteurs les rend difficilement constituables en une " classe " particulière, ce qui n'est pas sans poser des problèmes d'analyse. Pour ne donner que deux exemples, pour Adam \& Fayol (1989 : 3), les connecteurs/organisateurs, tout comme la ponctuation, s'opposent en priorité aux anaphores. Au contraire, Berrendonner \& Reichler-Béguelin (1989 : 123-124) rapprochent les connecteurs des anaphoriques, en observant notamment des similitudes dans leur gestion de l'enchaînement des clauses/phrases/propositions, tout en soulignant d'évidentes distinctions dans leur emploi syntaxique : ils observent effectivement certaines contraintes d'enchaînement similaires à celles régissant 
l'emploi des formes liées / formes de rappel dans le cadre de l'anaphore (Apothéloz, $1995: 18-25)^{1}$.

Ces dernières remarques nous interrogent particulièrement. En effet, que nous considérions les connecteurs en association des anaphores ou en opposition de cellesci, cela revient finalement toujours à les définir selon ces dernières, et ce qu'on aborde ces questions du point de vue syntaxique, du point de vue sémantico-référentiel ou encore du point de vue textuel; c'est d'ailleurs là un point commun aux études sur la question ( $c f$. bibliographie). Mais dans la mesure où l'emploi des anaphores a évolué dans le temps, et notamment dans le passage problématique de la phrase à la période (Goux, 2017 ; Goux 2019a ; Seguin, 1993 ; Siouffi, 2010), il devient pertinent d'inscrire l'étude des connecteurs dans une perspective diachronique. Particulièrement, et comme les bouleversements du fonctionnement des anaphoriques ont favorisé la constitution d'unités infratextuelles de plus en plus étroites (Siouffi, 2010), il convient d'étudier la façon dont les connecteurs ont accompagné ces changements au cours du temps afin d'en proposer une analyse approfondie, et de mieux saisir les paramètres accompagnant leur emploi. C'est ce que nous proposons au sein de notre contribution, qui viendra apporter des éléments de réflexion sur le rôle textuel des connecteurs au long du XVII ${ }^{\mathrm{e}}$ siècle.

Partant, et après avoir dans un premier temps défini textuellement les connecteurs que nous étudierons, nous présenterons notre corpus d'étude et les textes retenus pour nos analyses. Nous présenterons et analyserons les résultats obtenus tout d'abord en relation avec la ponctuation, ensuite en relation avec les anaphores, pour explorer et confronter les deux approches présentées plus haut ; enfin, nous comparerons rapidement nos résultats avec un second corpus de langue contemporaine pour vérifier la façon dont les paramètres étudiés évoluent en diachronie longue.

\section{Définition textuelle des connecteurs}

L'évolution générale de la fabrique de la textualité, au long de la période classique, est globalement celle d'un resserrement des unités infratextuelles autour d'une prédication principale, généralement entre des signes de ponctuation forts ou semiforts, alors que la tendance antérieure était davantage de proposer de longues unités de sens, consacrées au développement d'un topique de discours spécifique. Cette évolution fondamentale a entraîné avec elle une réanalyse et une reconfiguration de différents outils liés à la cohérence et à la cohésion textuelle (Ayres-Bennett \& Caron, 2016 ; Goux, 2019b : 305-309; Siouffi, 1997). Il serait ainsi surprenant que l'emploi des

\footnotetext{
${ }^{1}$ Une forme liée est une anaphore soumise à un contrôle syntaxique et qui n'est donc pas référentielle en elle-même, alors qu'une forme de rappel n'est pas soumise à un contrôle syntaxique et, partant, est pleinement référentielle. Ce type de contrainte est similaire, mutadis mutandis, à celui rencontré dans l'emploi de connecteurs argumentatifs comme car vs. parce que, qui s'appuie sur la connaissance partagée des locuteurs et la notion de connaissance a priori.
} 
connecteurs, et plus largement la notion de connexité, n'ait pas non plus subi de changements : une étude approfondie de l'évolution de la textualité ne peut, dans tous les cas, faire l'impasse de l'étude du changement subi par les connecteurs.

À notre connaissance cependant, aucun travail d'envergure n'a analysé l'évolution textuelle de ces connecteurs en diachronie, et ce bien qu'il y ait des études sur la grammaticalisation et/ou pragmaticalisation des connecteurs en français (Combettes, 1994 ; Rossari, 1998, entre autres). Même l'imposante Grammaire du français classique de Nathalie Fournier (2002) ne consacre pas de chapitre particulier à cette question et ce malgré sa sensibilité aux questions de textualité. Ainsi, bien que leurs métamorphoses morphologiques et morphosyntaxiques soient assez bien documentées, il reste à aborder les connecteurs du point de vue textuel en diachronie.

Comme il serait trop ambitieux de nous engager, pour cette contribution, dans une analyse exhaustive de tous les connecteurs envisageables en français (nous renvoyons, pour un essai de grammaire en langue contemporaine, à Rossari, 1998 ; à Rossari, 2002 ; et surtout à Rossari, Ricchi \& Wandel, 2018, pour se rendre compte de l'ampleur de la tâche), nous nous concentrerons sur un corpus restreint des différents connecteurs que l'on peut trouver en langue classique. Nous écarterons notamment de notre contribution les termes de liaison œuvrant spécifiquement à l'organisation thématique ou topicale du discours et les marqueurs métadiscursifs du type premièrement, deuxièmement..., dits aussi "syntagmes cadratifs" (Charolles \& Vigier, 2005) pour nous concentrer exclusivement sur la répartition commentée par les grammaires du temps : effectivement, les grammairiens et remarqueurs de l'époque classique avaient déjà repéré l'existence de ces connecteurs, quand bien même leur approche de la question serait plus modeste que les grandes tentatives contemporaines de classification. Le parcours de ces grammaires nous permettra cependant d'avoir une vision de la façon dont les locuteurs du temps abordaient cette question, d'évaluer les critères d'analyse qu'ils jugeaient pertinents dans cette problématique et, partant, de préparer la recherche de nos occurrences.

Il faut remarquer dans un premier temps que les doctes abordaient la notion de connecteur sous le terme générique de conjonctions. Leurs analyses se mêlent ainsi à celles des conjonctions de coordination et de subordination, au sens moderne du terme (Goux, 2019a : 109-110) : ainsi, nous pouvons trouver chez Chiflet (1659) et RégnierDesmarais (1706) les analyses suivantes, représentatives de la façon dont l'époque classique aborde les connecteurs.

Les conjonctions servent à lier les mots auec les mots, ou les phrases avec les phrases. De plus à passer d'vne periode à l'autre [...] Les vnes s'appellent Copulatives : comme, Et, außi \&c. Les autres Disjonctives: comme, $N y, O u$, Soit que \&c. Les autres Adsersatives, qui marquent quelque difference ou contrarieté : 
comme ; Mais, Toutefois, Neantmoins [...] (Chiflet, 1659: 122123).

La Conjonction est une Partie d'Oraison indéclinable, qui sert à la liaison des membres du Discours. [...] Tant \& que ne sont Conjonctions copulatives qu'estant employez relativement, comme dans cet exemple. Tant de part que d'autre, qui ne signifie autre chose que de part \& d'autre (Régnier-Desmarais, 1706 : 650-652).

Les doctes mettent ainsi en avant, et ce à l'instar des grammaires contemporaines (Grévisse \& Goosse, 2016 : 1421-1428 ; Riegel et al., 2014 : 104), deux propriétés fondamentales des connecteurs : (i) ce sont des termes de liaison, agissant soit au niveau du mot, soit au niveau du groupe du mots (ce que Chiflet, supra, appelle "phrase ", au sens classique du terme, $c f$. Seguin, 1993). Les connecteurs relient ainsi les " membres du discours ", des syntagmes, des GN, des propositions, des phrases voire des périodes entières ; (ii) ils peuvent être classés selon des critères argumentatifs et/ou rhétoriques.

Les grammaires contemporaines proposent souvent un classement assez fin de ces connecteurs, classement qui peut cependant devenir rapidement complexe à l'instar de ce que proposent Riegel et al. (2014 : 1043-1057). La GMF classe ainsi ces outils en connecteurs spatio-temporels, énumératifs, topicalisant, d'exemplification, de point de vue, explicatifs, reformulatifs, etc. Mais bien que ces catégories fassent sens, leur multiplicité les rend peu opérables et il n'est pas rare d'hésiter dans l'analyse, que ce soit pour des connecteurs très usuels dont les rôles sont, de fait, multiples et flottants (à l'instar de mais, qui peut être autant concessif qu'oppositif voire additif), tout comme pour des connecteurs très spécifiques, dont l'emploi s'accorde mal avec des catégories définies a priori. Au contraire, une classification par trop simplexe, à l'instar de celle que propose Le Bon Usage (Grévisse \& Goosse, 2016 : 1421-1428), conduit les auteurs à faire suivre la description générale de cinq pages " d'observations particulières ", tandis que les conjonctions de coordination à proprement parler (Grévisse \& Goosse, 2016 : 1509-1522), certes et sans doute car plus fréquentes en discours, font chacune l'objet d'une description approfondie, mais sans tentatives de classification ultérieure. Il nous semble alors que les grammaires du temps proposent une classification plus simple des connecteurs, que nous gagnons à reprendre par la suite pour nos analyses : nous y perdrons peut-être en grain, mais cela nous aidera à mieux déterminer les trajectoires d'emploi de ces connecteurs et cela nous empêchera de nous engager dans des descriptions par trop précises, incompatibles avec l'étude sur corpus que nous menons.

On trouve ainsi dans ces grammaires, généralement et selon les citations données précédemment, une tripartition du type copulation (addition), disjonction (alternative exclusive) et adversation (opposition). Pour les doctes, ces conjonctions / 
particules / connecteurs / termes de liaison permettent ainsi : (i) de construire une liste d'éléments, sans les hiérarchiser entre eux autrement que par la linéarité de l'énoncé (tels aussi, de plus, ensuite...) ; (ii) de proposer une alternative exclusive entre deux éléments ou plus d'une liste fermée, sans les hiérarchiser mais en les présentant comme équipollentes (tels d'une part / d'autre part, soit que, de part \& d'autre...) ; (iii) d'opposer deux éléments ou plus entre eux, cette fois-ci en les hiérarchisant, un élément se chargeant d'apporter une précision décisive empêchant ou minorant la réalisation particulière du premier membre argumentatif (tels néanmoins, cependant...).

On notera que ce qui permet de réunir ces différents outils, c'est ni leur identité catégorielle ou morphosyntaxique, puisque l'on va trouver dans ces listes des adverbes ou des groupes prépositionnels, avec des positions dans la période / la phrase / la proposition assez diverses ; ni leur rôle argumentatif ou rhétorique, puisqu'on explore différents opérateurs reconnus par la logique traditionnelle. Ce qui les réunit alors comme " classe ", c'est bien leur rôle organisationnel dans le cadre de l'énoncé, à l'aune de ce que nous observons dans les trois exemples suivants $(1 \text { à } 3)^{2}$ :

(1) Finalement il nous faut encores noter, qu'il est bien vray que ce premier iour de la sepmaine, au premier poinct de la clarté du iour auquel il pleut au Seigneur de ressusciter, nous represente le commencement de la lumiere du siecle à venir, comme il a esté dit : mais sachons cependant de sauoir si cela se feroit tout incontinent : \& au mesme instant qu'ils le voyoyent ressuscité, Act. 1. 6. Mais le maistre leur baille la response, laquelle seule nous doit resoudre contre toute impatience [...] (Bézé, 1593).

(2) [...] ce superflu est un levain contagieux à l'avenement d'une année abondante, qui corompt par un avilissement effroiable, toute matiere n'a guéres si précieuse, et pendant les effets si certains, et tant de fois marquez. Puis le haut prix à son tour, à sa revanche, et par l'abandonnement ou negligence de culture et prodigalité d'usage des grains, une année sterile faisant pancher la balance de l'autre côté [...] (Boisguilbert, 1695).

(3) Quand nous en serons couronnez, alors sçaurons-nous quelle elle est, mais tous les deux ne seront qu'apres cette vie. Pourtant quand moyse dit au Seigneur, ie te prie fay moy voir ta gloire, Exod. 33. 10. il luy dit, Tu ne pourras voir ma face, car l'homme ne me peut voir $\&$ viure apres cela. (Le Faucheur, 1632).

En (1) ainsi, cependant marque l'opposition entre la résurrection de Jésus d'une part, et le moment de l'évangile de l'autre, la concomitance entre les événements étant mise en question ; en (2), puis marque la succession temporelle, en plaçant l'événement décrit par la nouvelle proposition introduite dans la continuité de la précédente ; en

${ }^{2}$ Dans les exemples, nous soulignons en gras les connecteurs (et autres éléments) que nous commentons. Les références aux textes sont données sous la forme (Auteur, date) dans le corps de l'article, et en détail en bibliographie. 
(3) enfin, pourtant marque encore une fois une opposition, en présentant le propos introduit comme un argument minorant, ou contredisant l'information présentée précédemment. Chacun de ces connecteurs scinde ce faisant les énoncés en, au moins, deux parties qui s'articuleront de différentes façons entre elles et qui scanderont la lecture : on ne peut notamment interpréter parfaitement le segment introduit par les termes de liaison en gras sans prendre en compte le passage antécédent, et la compréhension de celui-ci dépend en retour directement du second qui vient minorer, contredire ou expliciter son sens. On notera également que ces connecteurs agissent au niveau supérieur au syntagme; ils articulent des prédications entre elles, quand bien même l'une d'entre elles seraient exprimées de façon elliptique, par exemple en ne répétant pas un verbe commun aux deux membres connectés (exemple 2 supra).

Partant, ce qui réunit ces termes au sein de la même famille, au moins aux yeux des doctes de l'époque classique, c'est leur rôle générique dans la structuration de l'énoncé et dans la fabrique de la textualité : il s'agit d'aider à construire le propos, en marge de l'évolution référentielle et de la prédication, en organisant le continuum textuel selon différentes strates sémantiques et argumentatives et en modulant la linéarité inhérente de l'énoncé, qu'il soit d'ailleurs tant écrit qu'oral. C'est ce paramètre qui permet de les rapprocher de la ponctuation, à l'instar de ce que défendaient Adam \& Fayol (1989) et de ce qu'expliquent Riegel et al. (2014, nous soulignons) :

Les connecteurs contribuent à une opération langagière fondamentale, la linéarisation. Les éléments linguistiques de différents niveaux sont intégrés dans un développement linéaire strict. [...] Les connecteurs, de même que la ponctuation, favorisent cette opération, en spécifiant les relations que les unités du texte entretiennent dans l'univers de croyance partagé entre locuteur et allocutaire ou proposé par le locuteur (Riegel et al., 2014 : 1057, les italiques sont nôtres).

La connexité, nous le rappelons cependant, ne présuppose pas nécessairement une hiérarchisation dans le sens strict du terme, puisque les éléments connectés peuvent se retrouver coplanaires, du moins sur un plan rhétorique, par exemple dans le cadre de l'addition. Quand bien même la linéarité de l'énoncé supposerait une consécution dans le cadre d'une liste, il n'y a pas nécessairement un élément " argumentativement " plus fort que l'autre : l'exception demeure, évidemment, l'opposition ou l'adversation, où l'on retrouve une opposition entre prémisse majeure et mineure, c'est-à-dire qu'un membre de l'énoncé influencera un précédent en lui apportant un bémol. Indépendamment des remarques typologiques dès lors, ces connecteurs peuvent être assimilés à des

[...] instructions données par l'énonciateur-destinateur à l'énonciataire-destinataire. Ils signalent qu'il y a lieu de calculer une relation sémantique entre deux propositions ou deux paquets de 
propositions successifs (paragraphes, parties de texte par exemple), ou entre des actes de langage [...]. Ils indiquent également quel type de relation il convient de calculer (Brassart, 1993 : 508).

Aussi, et en raisonnant en termes de grammaire de texte, si la cohésion se charge des liens inter-phrastiques par l'intermédiaire des phénomènes d'anaphore, et si la cohérence étudie la progression thématique, les connecteurs (et la connexité en général) agissent à un niveau plus diffus, plus localisé mais aussi et paradoxalement plus aléatoire : des études diverses ont notamment montré que la présence ou l'absence des connecteurs ne facilite pas nécessairement la compréhension ou la mémorisation des énoncés (Brassart, 1993 ; Corminboeuf, 2015 : 102). Quelque part, ces adjuvants ne font qu'expliciter une progression sémantico-rhétorique déjà explicitée par la construction même de l'énoncé, l'identité et la succession des référents, ou la nature des prédicats : les connecteurs seraient ainsi des indices contingents et non nécessaires au contraire, par exemple, de la continuité référentielle et de la progression thématique dont l'absence compromet nécessairement l'identité d'un texte en tant que tel. Comme le souligne, ainsi, Brassart :

Tout se passe comme si les adultes compétents compensaient, au moins en partie, l'absence des connecteurs par une série d'inférences relationnelles qui alourdissent les opérations de traitement on line, mais permettent la construction d'une représentation intégrée et des rappels libres qui ne se distinguent pas de ceux des versions connectées (Brassart, 1993 : 509).

Et Frank Neveu de préciser :

[...] contrairement à l'enchaînement anaphorique, [la connexité] ne saurait être tenue pour une condition nécessaire de la cohésion et de la cohérence des grandes masses verbales. Un texte peut être faiblement empreint de connexité sans que l'interprétabilité et la continuité informationnelle aient à en souffrir (Neveu, 2007 : 166).

Ce n'est pourtant pas parce que ces connecteurs sont contingents à la compréhension qu'ils doivent être négligés, tant l'information qu'ils apportent à la lecture peuvent cristalliser sa compréhension : et quand bien même n'auraient-ils pas subi, au cours de l'histoire de la langue, des bouleversements aussi notables que d'autres marqueurs de cohésion ou de cohérence, au-delà des phénomènes de grammaticalisation qui ont été étudiés, ils ont accompagné comme marque de connexité les changements de l'énoncé. Nous pouvons donc les prendre comme des témoins, plus que des causes ou des instruments, de l'évolution de la textualité à l'époque classique, et leur étude nous permettra de préciser, voire de nuancer, la chronologie de ces changements. 


\section{Constitution du corpus d'étude}

Nous avons choisi d'étudier ces problématiques par l'intermédiaire d'une étude sur corpus, plutôt que par l'étude d'une sélection d'occurrences. Deux raisons à cela : d'une part, et bien que l'interprétation sémantique de ces connecteurs soit relativement stabilisée en langue depuis l'époque classique comme nous l'indiquent les analyses des doctes $^{3}$, nous ne désirons pas nous rabattre sur notre seul sentiment linguistique pour analyser les occurrences; du moins, nous voulions la compléter par des données chiffrées, qui nous permettent d'atteindre une meilleure représentation des phénomènes que nous décrivons. D'autre part, nous voulions traiter un nombre significatif d'occurrences afin d'observer des dynamiques notables sur le siècle classique, notamment en relation avec les données accessibles dans d'autres grandes études sur la textualité au XVII ${ }^{e}$ siècle (Ayres-Bennett \& Caron, 2016 ; Goux, 2019b) et nous voulions confirmer si, comme nous en faisons l'hypothèse, les tendances d'emploi des connecteurs évoluent parallèlement à celles des marques anaphoriques.

La constitution de notre corpus d'étude n'a pas été sans difficulté, notamment à cause de notre ambition de relier l'étude des connecteurs à celle de la ponctuation. Effectivement, il fallait nous assurer que les occurrences que nous relevions n'avaient pas été " caviardées " par un éditeur ou un imprimeur ayant travaillé ultérieurement sur un texte de l'époque classique, modifiant, ajoutant ou supprimant des signes typographiques, ce qui aurait nécessairement perturbé les résultats que nous aurions pu obtenir. La base de données Frantext, sur laquelle nous nous sommes appuyé, nous permet de consulter les éditions utilisées pour sa constitution : nous nous sommes donc assuré que les textes dépouillés pour cette étude étaient des éditions originales ${ }^{4}$. Nous rappelons en passant que nous ne pouvons nous prononcer sur l'origine véritable de la ponctuation de ces textes : à l'époque classique, ces choix étaient généralement laissés à discrétion des éditeurs ou imprimeurs, les auteurs y accordant rarement de l'importance avant le XVIII siècle (Demonet, 2011 : 138). Nous avons donc considéré comme relevant de la "ponctuation originale " celle de la première édition datée d'une ouvre spécifique, considérée comme telle par Frantext, sans nous prononcer quant à la main véritable qui la prit en charge, auteur, éditeur ou imprimeur. Nous avons cependant

\footnotetext{
${ }^{3}$ On notera que certains connecteurs, et notamment ceux issus de phénomènes de grammaticalisation comme néanmoins ou cependant peuvent parfois se rencontrer en français préclassique dans leurs emplois archaïques, notamment temporels. Ces emplois sont cependant condamnés par les remarqueurs et ne sont pas représentatifs des tendances de la langue du temps.

${ }^{4}$ Nous n'avons pas pu cependant, compte tenu de leur grand nombre, vérifier manuellement et systématiquement la concordance entre la numérisation de Frantext et les éditions princeps de toutes nos occurrences. Les vérifications manuelles opérées sur environ deux tiers des occurrences n'ont pas fait émerger de différences, mais nous demeurons cependant prudent quant à l'exactitude parfaite de nos résultats malgré la confiance que nous portons aux équipes de l'ATILF (UMR 7118, Université de Lorraine).
} 
considéré que notre étude, portant davantage sur des pratiques linguistiques que sur des tendances stylistiques, pouvait faire l'impasse sur cette distinction sans compromettre la validité de nos résultats.

Ce choix méthodologique a eu, néanmoins, des conséquences directes sur les connecteurs que nous avons analysés dans la mesure où pour certains d'entre eux, les éditions princeps présentes sur Frantext au moment de notre recherche ne nous permettait pas de relever suffisamment d'occurrences pour avoir des résultats interprétables. Nous avons alors décidé de resserrer notre exploration à quelques connecteurs uniquement, en espérant que de nouveaux corpus permettront à l'avenir d'explorer d'autres termes de liaison. Partant, nous ne proposerons ici qu'une étude de deux connecteurs additifs, puis et ensuite, et de trois connecteurs adversatifs, cependant, néan(t)moins et pourtant. Initialement, nous souhaitions explorer également les connecteurs disjonctifs et notamment le couple d'une part / d'autre part mais nous ne trouvions, dans les textes de Frantext correspondant à nos critères de recherche, que 4 et 12 occurrences respectivement, contre des milliers pour les autres connecteurs. Cela rendait dès lors toute étude, quantitative comme qualitative, de ces connecteurs impossible : nous avons alors choisi de délaisser cette partie de notre travail, du moins dans le cadre de cette contribution.

Le corpus retenu compte 600 occurrences des cinq connecteurs donnés plus haut, relevés dans des textes publiés sur la période 1580-1720. Comme il est souvent difficile de segmenter linguistiquement la période classique ( $c f$. Ayres-Bennett \& Caron, 2016 : 339-345; Combettes, 2003 ; Goux, 2019b : 71-72), nous avons repris pour ce paramètre temporel une découpe chronologique traditionnelle allant de 1600 à 1700, que nous avons légèrement étendue de vingt ans en amont, et de vingt ans en aval, afin d'adoucir les bornes de cette période de l'évolution de la langue française et de mieux saisir la dynamique de l'évolution. Les connecteurs retenus pour cette étude se rencontrent au sein d'un ensemble de textes génériquement variés, du texte narratif à la poésie en passant par l'essai philosophique ou religieux. Pour homogénéiser nos remarques, nous avons choisi de nous orienter exclusivement vers des textes appartenant à des domaines liés à la narration et à l'argumentation, qui sont les lieux privilégiés de l'emploi des connecteurs. Nous rappelons en passant que les chercheurs (par exemple Adam, 1987), pour essayer de définir linguistiquement les genres textuels ou, du moins, pour proposer une typologie de leurs séquences, prêtent une attention particulière à ces connecteurs. Cela nous a alors conduit à éliminer de notre corpus les textes versifiés, théâtre et poésie, dont la perspective stylistique d'une part, et les contraintes d'écriture de l'autre, auraient déstabilisé nos analyses.

Bien que ce paramètre générique ait orienté la constitution de notre corpus, nous n'y ferons cependant référence que très ponctuellement au long de notre développement : nous nous concentrerons effectivement surtout sur la dimension microtextuelle, en analysant la place de ces connecteurs au sein des unités phrastiques et 
périodiques, en relation avec la ponctuation et la dynamique référentielle des énoncés, et l'évolution de ces paramètres au long de l'époque classique. Les relevés que nous présenterons ne tiendront donc pas compte du genre de textes. De plus, et compte tenu de l'écart qu'il peut exister entre les catégories enregistrées par les bases de données et le paysage littéraire du temps, une analyse générique en tant que telle ne peut être que réductrice et incomplète. Il faudrait effectivement la compléter d'une analyse des séquences textuelles pour avoir des résultats concluants ( $c f$. Goux, 2019b : 245-266) ; or, ce type d'analyse étant particulièrement long, il excède les dimensions de notre contribution, raison pour laquelle nous avons choisi de la mettre de côté.

Nous présentons ci-dessous les résultats de notre exploration de Frantext. À partir d'une recherche exhaustive des connecteurs retenus, nous avons sélectionné une partie de ces occurrences en veillant à observer une progression chronologique régulière au long du temps et à varier, autant que faire se pouvait, les textes dont les occurrences sont issues. Pour équilibrer notre corpus de travail, nous nous sommes arrêté sur le chiffre arbitraire de 200 occurrences par connecteur; cela représente, comme nous le voyons ci-dessous, entre 14 et $40 \%$ des occurrences totales de chaque connecteur relevé dans Frantext. Dans la mesure où nous devions, pour notre étude, approfondir l'analyse de nos occurrences au-delà de l'emploi de la ponctuation, notamment en observant ce qu'il se passait au niveau de la dynamique informationnelle et du sens des énoncés, il nous a nécessairement fallu restreindre ce corpus.

\begin{tabular}{|c|c|c|c|c|c|}
\cline { 2 - 6 } \multicolumn{1}{c|}{} & \multicolumn{2}{c|}{ Connecteurs additifs } & \multicolumn{3}{c|}{ Connecteurs adversatifs } \\
\hline $\begin{array}{c}\text { Connecteur } \\
\text { (lemme) }\end{array}$ & Puis & Ensuite & Cependant & Néanmoins & Pourtant \\
\hline $\begin{array}{c}\text { Occurrences } \\
\text { totales }\end{array}$ & 1350 & 757 & 1370 & 1124 & 471 \\
\hline $\begin{array}{c}\text { Occurrences } \\
\text { sélectionnées }\end{array}$ & 200 & 200 & 200 & 200 & 200 \\
\hline $\begin{array}{c}\text { Représentativité } \\
\text { de notre corpus }\end{array}$ & $14.8 \%$ & $26.4 \%$ & $14.6 \%$ & $17.8 \%$ & $42.4 \%$ \\
\hline
\end{tabular}

Tableau 1. Constitution du corpus d'étude.

Pour circonscrire le rôle des connecteurs dans la fabrique de l'énoncé, nous avons par la suite analysé deux paramètres en particulier : (i) la ponctuation directement employée en amont et en aval de ce dernier; (ii) son rôle dans la dynamique périodique. Pour ce critère, nous avons dû développer une stratégie nous permettant de traiter efficacement un grand nombre d'occurrences, sans rentrer dans les détails d'une analyse générique ou séquentielle, ou qui nous aurait demandé de prendre en compte un co(n)texte interprétatif d'une grande densité. Nous avons alors surtout observé si l'emploi du connecteur était concomitant soit avec un rappel (pro)nominal d'un référent antérieur, ce qui traduit, pour le locuteur, la nécessité de réinitialiser ou de poursuivre une chaîne de coréférence après une parenthèse ou une digression 
périodique trop longue (4); soit avec l'apparition d'un nouveau référent, généralement en fonction sujet, qui signale l'introduction d'un nouveau mouvement périodique (5) :

(4) [...] nostre Esprit par le moyen de la volonté, c'est à dire, de cette force qu'il a de se mouuoir $\&$ de se déterminer, s'arreste, $\&$ suspend son jugement, s'il y void quelque obscurité, ensuite il delibere, \& si tost qu'il y remarque quelque chose de clair [...] (La Forge, 1661).

(5) J'assemblay promptement nos Suisses, que je mis en bataille pour m'opposer audit duc de mayenne, s'il vouloit passer plus outre vers Laon. Les sieurs de Vic et de sainct denis Mailloc se joignirent incontinent à moy. Monsieur de Nevers y vint bien tost apres, puis le roy y arriva qui loua grandement le devoir que j'avois fait d'opposer les Suisses au passage dudit duc [...] (Harlay de Sancy, 1610).

Analyser la progression périodique, cette " toise élastique ", sous ce seul paramètre, est nécessairement réducteur, tant nous savons à quel point la période est d'une définition compliquée. Ne serait-ce, les chercheurs sont encore loin de s'accorder sur les bornes à donner à cette unité textuelle, que ce soit pour la période classique (Siouffi, 2010) ou contemporaine (Berrendonner et al., 2012). Dans ce dernier ouvrage par exemple, après un parcours quasi-complet de toutes les dimensions envisageables de la période, les auteurs reviennent finalement à Aristote (Berrendonner et al., 2012 : 348) par défaut de produire une définition uniforme de cette unité, qui satisferait les nombreux paramètres (morphosyntaxiques, prosodiques, argumentatifs, textuels...) qu'il conviendrait de prendre en compte. Si nous considérons cependant que le développement périodique est lié avant toutes choses à la progression d'un topique de discours comme le propose Pierre Le Goffic (2011 : 22 sq.), la prise en compte de la continuité et de la succession référentielle permet d'avoir une idée, même approximative, de la dimension de la période au sein d'un énoncé ; du moins, cela nous est suffisant pour étudier le rôle des connecteurs et de déterminer à quel niveau ils interviennent, infrapériodique (4) ou inter-périodique (5).

\section{Ponctuation et connecteurs}

\subsection{Tendances générales}

Dans un premier temps, nous proposons une analyse des signes de ponctuation rencontrés dans ce corpus en association avec les connecteurs retenus. Nous avons distingué, pour chaque occurrence, le symbole employé directement à gauche, et directement à droite du connecteur. Nous avons organisé nos résultats par tableaux, en mettant en avant, par des italiques, les occurrences les plus notables de chaque catégorie. 


\begin{tabular}{|c|c|c|c|c|c|c|c|}
\hline & \multicolumn{3}{|c|}{ Puis } & \multicolumn{4}{|c|}{ Ensuite } \\
\hline À gauche & , & ; & . & , & ; & : & . \\
\hline Occurrences & 54 & 20 & 20 & 10 & 7 & 6 & 16 \\
\hline À droite & , & & & , & ; & : & . \\
\hline Occurrences & 18 & & & 28 & 1 & 6 & 1 \\
\hline
\end{tabular}

Tableau 2. Ponctuation et connecteurs additifs.

\begin{tabular}{|c|c|c|c|c|c|c|c|c|c|c|c|c|}
\hline & \multicolumn{5}{|c|}{ Cependant } & \multicolumn{4}{|c|}{ Néanmoins } & \multicolumn{3}{|c|}{ Pourtant } \\
\hline À gauche & , & ; & $:$ & $?$ & . & , & ; & $!$ & . & , & ; & . \\
\hline Occurrences & 10 & 10 & 7 & 2 & 65 & 10 & 12 & 1 & 12 & 8 & 1 & 19 \\
\hline À droite & , & & & & & , & & & & , & ; & . \\
\hline Occurrences & 26 & & & & & 11 & & & & 17 & 2 & 2 \\
\hline
\end{tabular}

Tableau 3. Ponctuation et connecteurs adversatifs.

Nos relevés montrent, dans un premier temps, que la ponctuation est rarement employée avec ces connecteurs. L'adverbe additif puis est sans doute la forme qui se démarque le plus de notre corpus, puisqu'environ un quart des occurrences relevées est précédée d'une virgule, ce qui traduirait ainsi le nouvel élan dans l'énumération ou le propos qui accompagne l'emploi de l'adverbe. Nous comparerons cela avec le connecteur ensuite, rarement précédé d'une virgule dans ce corpus mais davantage par un point. Cette différence dans la segmentation des énoncés nous conduit à envisager qu'il est une différence entre ces deux connecteurs additifs, pourtant synonymiques ou quasi-synonymiques, tant en langue classique qu'en langue moderne : du moins, ni les grammaires contemporaines consultées (Grévisse \& Goosse, 2016 ; Riegel et al., 2014), ni les grammaires du temps accessibles dans les corpus numériques en ligne ${ }^{5}$, ne les commentent en détail, les rassemblant dans la même liste de "connecteurs additifs". La nuance se situerait ainsi au niveau de l'unité que relient ces connecteurs : puis aurait davantage un rôle au niveau propositionnel ou périodique, tandis qu'ensuite interviendrait davantage au niveau de la prédication elle-même. C'est ce qu'illustreraient les exemples suivants :

(6) Comme si l'on dit, par exemple, qu'entre les adjoincts de l'homme les uns sont du corps, les autres de l'esprit ; que ceux du corps sont la taille, la force, la santé, la beauté, etc. Ceux de l'esprit, diverses facultez, et les habitudes des arts, des sciences, des vertus dont on fasse ensuite le denombrement. Nous avons indiqué plus haut que les adjoincts sont ce que plusieurs apellent accidens, et qu'ils peuvent aussi estre appellez qualitez [...] (Bernier, 1684).

(7) [...] Et ils ne manquent jamais de se procurer cette situation de surcroi, toutes les fois que les grains rencherissent, quand ce n'est point dans l'excés, puis quand ils viennent à baisser, on peut dire que les laboureurs sont ruïnez, ainsi que toutes les professions qui en attendent leur destinée, et qu'ils perdent

${ }_{5}$ À l'instar du corpus des grammaires Garnier Numérique (Colombat et al., 2011). 
dans la suite ce qu'ils ont gagné dans les précedentes années [...] (Boisguilbert, 1695).

En (6) ainsi, le développement initié par ceux de l'esprit ${ }^{6}$ s'étend au long de la phrase graphique considérée et compose une période en elle-même, consacrée à ce topique de discours. Le retour à la P4 Nous dans la phrase subséquente marque effectivement un nouveau mouvement périodique par la remise en avant d'un nouveau référent, ici l'auteur du texte, qui intervient dans le propos pour introduire un commentaire métalinguistique sur sa terminologie : cela vient rompre alors le développement thématique initié précédemment. Ensuite agit dès lors au sein du dernier membre de la première période, et a une sorte de rôle conclusif : du moins, il enrichit cette interprétation en préparant, en quelque sorte, la clôture périodique qui est prise en charge par l'évolution du référence sujet et du thème de l'énoncé.

En revanche, dans l'exemple (7), puis poursuit la période initiée consacrée aux grains par l'intermédiaire du pronom ils: l'auteur prolonge donc le développement du même topique de discours, comme l'indique la chaîne anaphorique qu'il poursuit par l'intermédiaire de cette forme anaphorique liée. Puis introduit ce faisant un nouveau membre périodique et l'articule avec le précédent, qui faisait office de conclusion intermédiaire par l'intermédiaire de la conjonction et en tête d'exemple (7). Le connecteur a ainsi un rôle de premier plan dans la fabrique de la textualité et participe à l'introduction de ce nouveau membre périodique, créant comme un nouvel élan dans l'énoncé ou une relance de ce dernier ; ou plutôt et en accord avec ce que nous disions précédemment sur le rôle contingent des connecteurs, il vient renforcer ou anticiper cette interprétation que prend surtout en charge le pronom anaphorique $i l s$, dont l'interprétation exige de prendre en compte le continuum textuel à sa gauche dans un mouvement transpropositionnel.

Si ce n'est le cas particulier de puis cependant, nous n'observons dans notre corpus que peu de démarcation graphique autour des connecteurs que nous avons sélectionnés. Nous savons, par l'intermédiaire d'études sur la question (voir, notamment, l'ouvrage collectif dirigé par Dauvois \& Dürrenmatt, 2011), que l'emploi de la ponctuation s'est progressivement stabilisé, en tant qu'aide à la lecture silencieuse, au long de l'époque classique et permettait de faciliter en particulier l'interprétation de l'énoncé en isolant les différents groupes syntaxiques. Comme le soulignait Demonet (2011: 137), la typographie marque alors surtout, au long des $\mathrm{XVI}^{\mathrm{e}}$ et $\mathrm{XVII}^{\mathrm{e}}$ siècles, " ce qui n'est pas spontané dans la lecture orale : les parties du discours, les membres de la phrase, les rections (sujet/verbe) et les flexions (singulier/pluriel) ». Dans la mesure où les connecteurs que nous avons étudiés sont assimilables à des compléments de phrases, ils n'ont pas de rôle syntaxique à proprement parler, du moins, pas au sein des

\footnotetext{
${ }^{6}$ Le pronom démonstratif reprend les « adjoincts », concept philosophique développé par l'auteur et proche du concept de qualité.
} 
prédications qu'ils introduisent ou dans lesquelles ils s'insèrent : ils n'occupent pas de fonction sujet ou de compléments d'objets, et ils ne rentrent pas dans la structure de tel ou tel syntagme, étant comme flottants dans les énoncés. Même si l'on observe certaines contraintes positionnelles, à l'instar de puis qui sera toujours en tête du membre argumentatif qu'il introduit, ces connecteurs se caractérisent surtout par une certaine flexibilité : ensuite, ne serait-ce, peut autant se retrouver en tête de membre argumentatif qu'en son sein ( $c f$. exemples 4 et 6 ), les nuances s'opérant davantage au niveau rythmique ou stylistique qu'au niveau purement syntaxique ou textuel.

Comme les connecteurs ont néanmoins un rôle sémantique et argumentatif/rhétorique important dans la compréhension de l'énoncé, on peut s'attendre à ce que leur isolement typographique soit plus prononcé au fur et à mesure du temps, à l'instar de qu'observait Demonet (2011 : 137), notamment pour signaler leur détachement syntaxique avec le contexte gauche et le contexte droit et, partant, pour les mettre en valeur au cours de la lecture et signaler qu'il convient de les prendre en compte dans la compréhension. Comme le peu d'occurrences de ponctuation relevées nous empêche, au-delà de l'opposition entre puis et ensuite, de commenter les tendances générales enregistrées au long du siècle, nous avons exploré cette problématique en relation avec le paramètre temporel, avec de meilleurs résultats.

\subsection{Tendances diachroniques}

L'évolution de la ponctuation entourant ces connecteurs au long du temps dessine effectivement des tendances qui semblent confirmer notre dernière hypothèse : globalement, l'on observe effectivement de plus en plus d'isolement typographique des connecteurs dans les énoncés, quelque ponctuation que nous prenions en compte et ce selon des proportions dont les tableaux 2 et 3 permettent de rendre compte. Cet isolement typographique est cependant à nuancer en fonction des connecteurs étudiés : effectivement, si nous mettons en regard ces différents résultats avec une évolution diachronique, les résultats que nous obtenons sont loin d'être uniformes.

Pour mieux rendre compte des phénomènes, nous avons choisi de représenter nos résultats sous forme de graphiques : dans ceux-ci, nous avons indexé la progression chronologique en abscisse. Chaque ligne verticale représente une occurrence " positive " de ponctuation sur cet axe, qu' elle se situe directement à sa gauche ou à sa droite ou que le connecteur soit parfaitement encadré par la ponctuation ; une occurrence sans ponctuation d'aucune sorte ne produit quant à elle pas de barre. Enfin, la largeur de la barre donne une estimation de l'intensité du phénomène sur une date donnée. $\mathrm{Ce}$ " code barre " permet dès lors de voir aisément la progression diachronique de l'emploi de la ponctuation autour de ces connecteurs, et de mesurer leur évolution. 
(i) Connecteurs additifs :

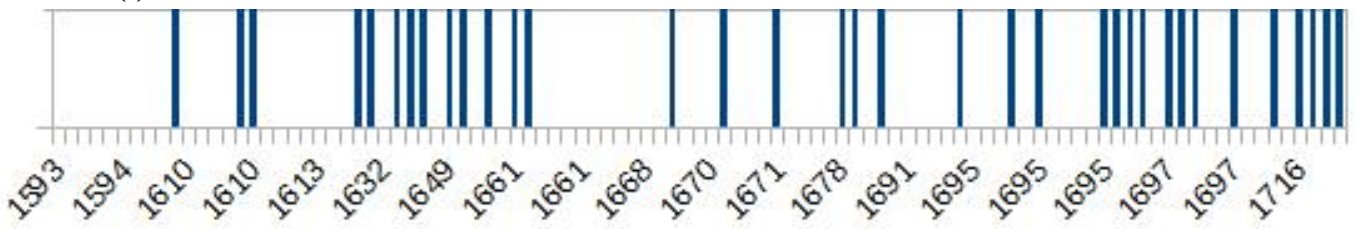

Figure 1. Ponctuation et puis.

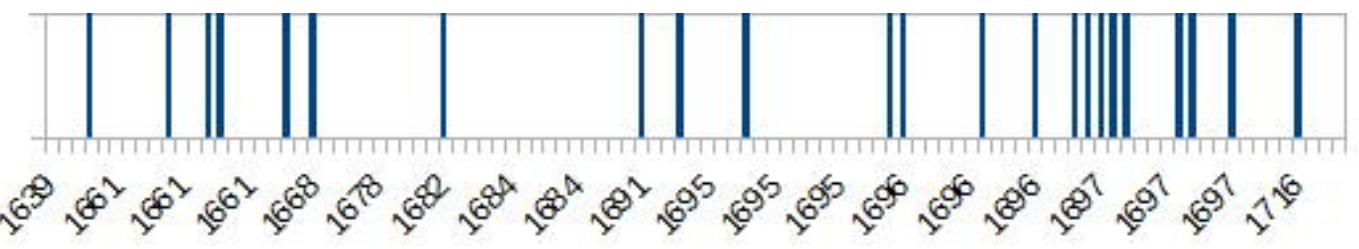

Figure 2. Ponctuation et ensuite.

(ii) Connecteurs adversatifs :

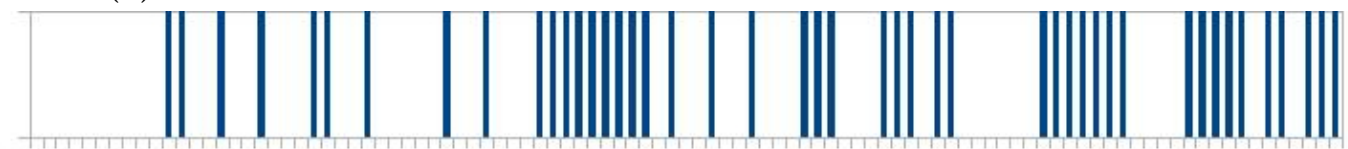

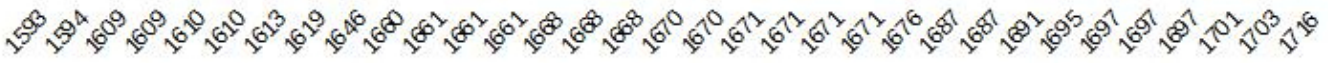

Figure 3. Ponctuation et cependant.

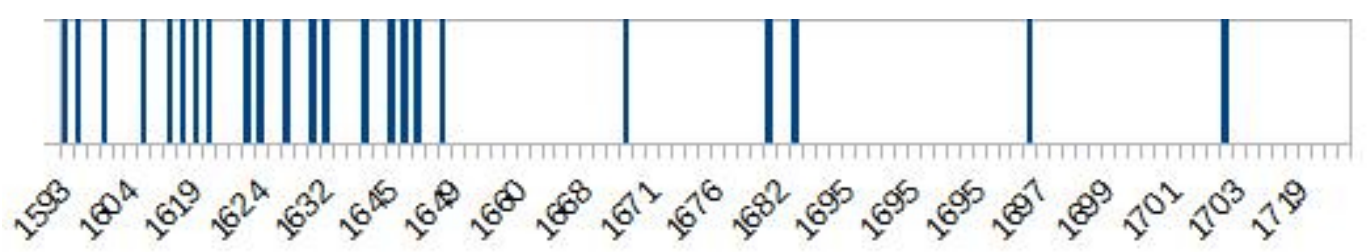

Figure 4. Ponctuation et pourtant.

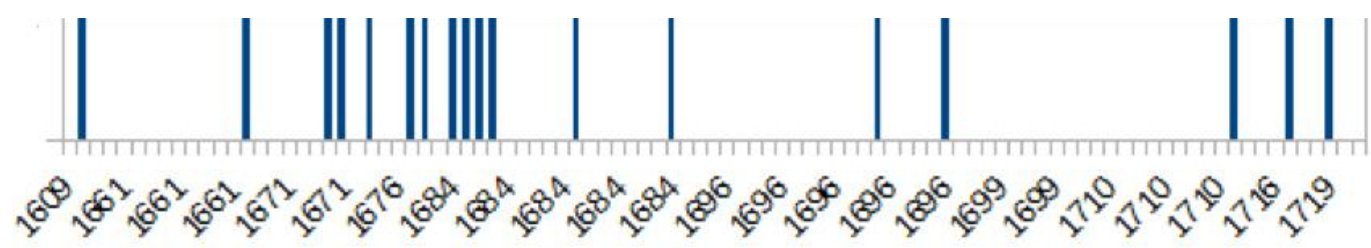

Figure 5. Ponctuation et néanmoins.

Si ce n'est les exemples intrigants de pourtant et de néanmoins, qui vont à l'encontre des prédictions théoriques et que nous commenterons dans notre prochaine partie, les connecteurs de notre corpus se révèlent bien être de plus en plus isolés par la ponctuation au fur et à mesure du temps. Cela traduit notamment une connaissance plus aiguë du code de la typographie, qui évolue sensiblement d'une aide à la lecture orale à une ponctuation davantage syntaxique, destinée à isoler dans le continuum textuel les éléments, à l'instar de ces différents connecteurs, qui n'ont pas de conséquence sur la syntaxe de l'énoncé et ce comme l'évoque la citation de Demonet (2011 : 137) 
donnée plus haut. Cet isolement a également d'autres conséquences, notamment culturelles et sémantiques, liées à la façon dont l'écrit était considéré à l'époque. Comme le rappelle également l'autrice :

[...] l'écrit acquiert une autorité qui dépasse désormais celle de la conversation spontanée et du témoignage direct (au milieu du $\left[\mathrm{XVI}^{\mathrm{e}}\right]$ siècle en France), la parole primaire est considérée comme un au-dessous du texte, distincte du code écrit (Demonet, 2011: 135).

Isoler davantage ces connecteurs, c'est ainsi proposer un système de segmentation qui se superpose d'abord, se substitue ensuite, aux réalités de la langue parlée. Il ne s'agit donc pas pour les compositeurs (auteurs ou éditeurs, en fonction du texte) de retranscrire une prononciation, réelle ou supposée, de la parole humaine, mais de proposer un nouveau système d'interprétation. Comme « [l]a question de la frontalité est [...] liée à la problématique des observables graphiques » (Neveu, 2007 : 175), il nous faut alors envisager que la ponctuation a également une conséquence sémantique, du moins sur la compréhension du propos. Le détachement crée une mise en lumière et provoque une attention particulière. Isoler, c'est avant tout individualiser et, partant, accorder une importance particulière à l'élément en question : le geste vient dès lors s'ajouter à l'interprétation première de certains connecteurs. Les compositeurs peuvent ainsi, en (ré)investissant ces nouveaux signes de ponctuation, moduler finement les interprétations dans l'emploi d'un même connecteur et enrichir considérablement leurs possibilités d'emploi. La comparaison entre les deux exemples suivants le fera mieux voir :

(8) Les occasions de sterilité qui dependent de la part de la femme seront aussi declarees par leurs signes: mais cependant faut remarquer, suyuant l'aduertissement d'aristote au premier liure de la generation des animaux, qu'il $\mathrm{y}$ a quelques femmes, lesquelles ont cela de leur disposition naturelle, qu'elles ne conçoiuent point en leur aage premiere, mais seulement en leur seconde $\&$ tierce [...] (Marinello, 1609).

(9) Le nom de Roi étoit odieux. Celui à qui S. Pierre le donne, étoit l'Empereur Néron, qui regnoit dans ce temps-là ; C'est-à-dire, le Tyran le plus cruel qui fut jamais; un Monstre; l'Horreur de tout le Genre Humain.

Cependant, dit l'apotre, c'est ce Roi méme, dont le regne est si cruel, la persoñe si odieuse, que vous devés honnorer (Rossal, 1701).

L'emploi argumentatif de cependant, dans ces deux occurrences, est du même ordre : conformément à l'instruction propre à ce connecteur adversatif, il s'agit de marquer une opposition et un nouveau mouvement périodique, par l'introduction de nouveaux référents : quelques femmes en (8), Néron [ce Roi] en (9), qui passe du statut d'attribut à celui de sujet par le biais d'une promotion syntaxique qui le consacre, effectivement, en nouveau référent ancrant le début d'un nouveau développement 
périodique. On remarquera cependant qu'en (8), il faut un redoublement argumentatif par l'intermédiaire de la conjonction mais pour marquer davantage l'opposition, là où la concomitance entre l'emploi du connecteur cependant et le début de phrase graphique permet d'attirer l'attention du lecteur sur ce nouveau mouvement argumentatif dans l'exemple (9). Autrement dit, la ponctuation forte du point simple, ou encore la segmentation graphique, se fait équivalente, mutatis mutandis, à la conjonction de coordination. En faisant endosser la charge de ce nouveau mouvement argumentatif non par le connecteur seul mais par l'emploi conjoint de celui-ci avec la ponctuation, la liberté offerte aux compositeurs se fait plus grande. Comme nous le présagions cependant, il ne s'agit pas tant d'une innovation que d'une reconfiguration, les grands mouvements du changement s'opérant davantage dans la dynamique informationnelle et la succession référentielle, par exemple dans l'emploi du pronom relatif lesquelles (exemple 8, cf. Goux, 2019b) ou l'inversion sujet-verbe, qui devient marginale au début du français préclassique si ce n'est, par exemple, dans des cas d'incise (exemple 9, $c f$. Prévost, 2001 : 12-19).

Comme nous l'avons vu cependant plus haut, deux de nos connecteurs ne suivent pas cette tendance générale et observable empiriquement : pourtant et néanmoins. Même, leur tendance est au parfait inverse de ce que l'on peut observer dans nos autres occurrences ; et de plus, ces connecteurs font partie de la famille des "adversatifs ", au même titre que cependant qui relève de la tendance générale : on ne saurait donc s'arrêter sur ce paramètre sémantique exclusivement. S’il semble bien y avoir une reconfiguration quant au rôle de la ponctuation avec ces connecteurs en particulier, l'explication que nous avons proposée, en termes de segmentation et de mise en lumière de membre de l'énoncé, n'est pas satisfaisante : il nous faut donc envisager une étude plus approfondie de nos connecteurs, cette fois-ci selon des paramètres liés à la progression périodique.

\section{Du reposoir à la structure}

\subsection{Connecteurs et dynamique référentielle}

Pour étudier cet aspect de l'emploi des connecteurs, nous avons repris le paramètre donné précédemment ( $\$ 2$, exemples 4 et 5$)$, et nous avons repris le même mode de représentation graphique. Une barre verticale indique, cette fois-ci, l'apparition d'un nouveau référent, et donc l'initiation d'un nouveau mouvement périodique ${ }^{7}$; et une

\footnotetext{
${ }^{7}$ Nous précisons ici que par «nouveau référent ", nous ne voulons pas dire que celui apparaissait pour la première fois à ce moment-là du développement de l'énoncé et n'a jamais été abordé dans le texte en question. Il est « nouveau " du point de vue micro-textuel, ou macro-syntaxique, dans la mesure où il n'était pas le topique de l'énoncé antécédent ; mais il a pu être évoqué auparavant, explicitement ou non. Une fois encore, nous sommes conscient de la limite de notre stratégie exploratoire, mais cela nous permet de tracer déjà quelques lignes de force.
} 
fois encore, la largeur de la barre témoigne, sur une période temporelle donnée, de la vivacité du phénomène.

(i) Connecteurs additifs :

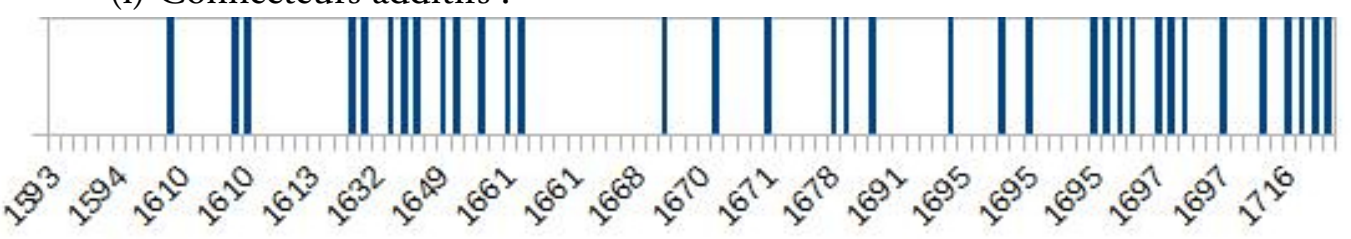

Figure 6. Puis concomitant à un nouveau référent.

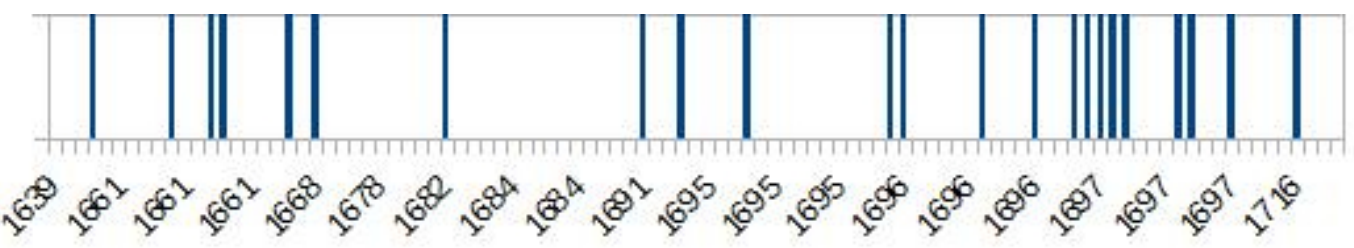

Figure 7. Ensuite concomitant à un nouveau référent.

(ii) Connecteurs adversatifs :

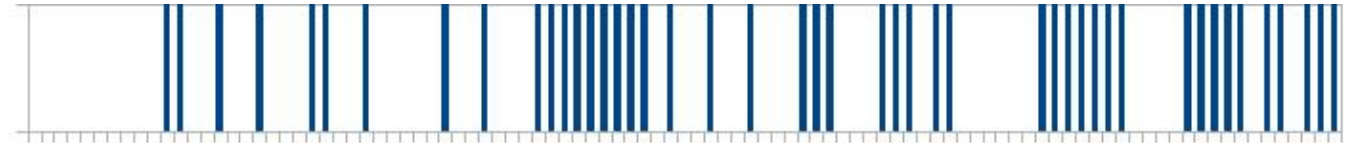

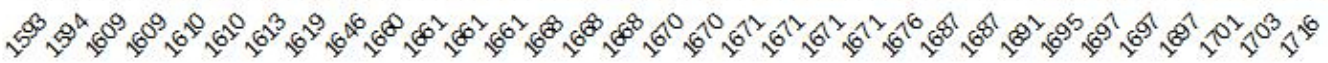

Figure 8. Cependant concomitant à un nouveau référent.
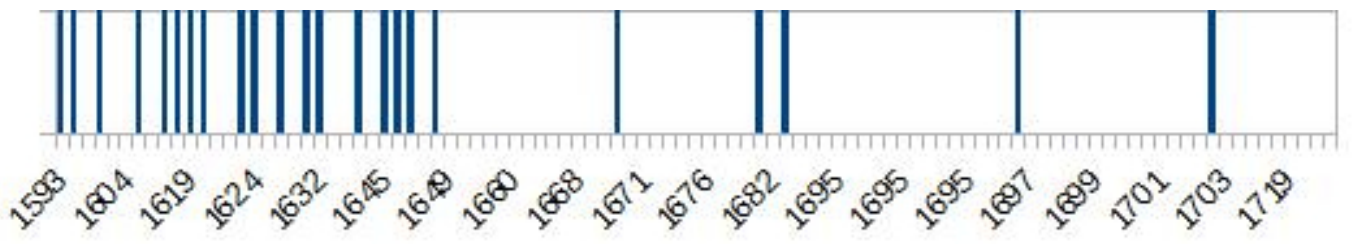

Figure 9. Pourtant concomitant à un nouveau référent.

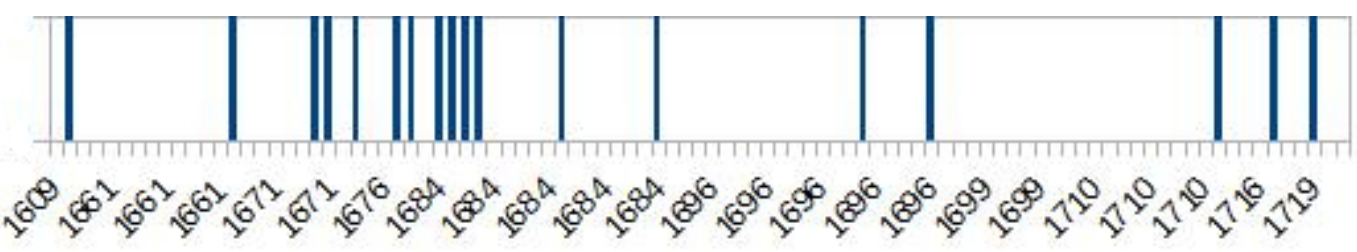

Figure 10. Néanmoins concomitant à un nouveau référent.

La comparaison de ces résultats avec les précédents ( $c f$. figures 1 à 5) nous permet de tracer une relation directe entre la ponctuation d'une part, et la progression de l'énoncé de l'autre, du moins selon les paramètres que nous avons choisi d'explorer. Cela nous permet également d'éclairer l'étrangeté que nous relevions auparavant, et le 
rôle apparemment distinct de cependant au sein de notre corpus au regard des autres connecteurs adversatifs étudiés.

Comme nous en faisions l'hypothèse, cependant s'établit de plus en plus au long de la période classique comme un marqueur adversatif de premier plan, à l'emploi concomitant de l'apparition d'un nouveau référent, ce qui est cohérent avec l'ultime phase de grammaticalisation, ou de pragmaticalisation de l'adverbe, qui évolue d'un marqueur temporel à un connecteur argumentatif (voir, par exemple, Combettes, 1994). Cette réanalyse comme marqueur adversatif s'est donc accompagnée dans les textes, d'une part, par un meilleur isolement typographique, l'isolation permise par la segmentation lui permettant d'augmenter son " poids " argumentatif, du moins de rendre inévitable sa prise en compte lors de la lecture ; et d'autre part, par son emploi de plus en plus privilégié avec le développement d'un nouveau topique de discours, l'association des deux phénomènes rendant ce nouvel élan périodique des plus repérables dans le continuum textuel ${ }^{8}$.

Au contraire de cependant, et si nous nous focalisons sur les mêmes paramètres, pourtant et néanmoins tendent en revanche à davantage s'inclure au sein du propos et marquent dès lors un argument moins prononcé, une adversation moins marquée dans l'argumentation. Il convient dès lors de mesurer, voire de nuancer les analyses qui établiraient une directionnalité à la pratique de la ponctuation en français, et qui traceraient univoquement une évolution d'une ponctuation servant de "guide à la lecture " à une ponctuation davantage syntaxique, isolant les syntagmes et épousant les diverses relations de dépendance ( $c f$. Rault, 2020 : 324-327, notamment par l'intermédiaire de la "grammaticalisation du point"). On aurait pu notamment s'attendre à ce que des éléments qui ne dépendent pas de la valence verbale, et qui ont une position relativement libre dans l'énoncé, soient de plus en plus encadrés par la ponctuation, par des virgules par exemple, pour mettre en lumière leur indépendance ${ }^{9}$ syntaxique. Plus que d'autres, ces connecteurs adverbiaux sont des candidats idéaux pour ce type de segmentation : le panorama que nous avons observé est cependant plus nuancé, l'emploi de la ponctuation n'étant pas uniforme dans le temps avec tous les connecteurs étudiés.

En réalité, la ponctuation encadrant ces connecteurs, et nos occurrences de pourtant et néanmoins le montrent, est également liée à l'importance argumentative de ceux-ci et à la dynamique informationnelle des énoncés; tant et si bien que même si nous prenons des occurrences encadrées par au moins un signe de ponctuation, l'on peut aisément voir que leur force argumentative est sensiblement distincte au long du

\footnotetext{
${ }^{8}$ Une étude complémentaire mériterait d'être faite avec la découpe en paragraphe, qui participe évidemment à cette opération de segmentation dans le texte en améliorant la "vi-lisibilité » sur l'espace de la page. Il faudrait notamment observer si Cependant se trouve davantage en début de paragraphe que pourtant et néanmoins, le paragraphe s'installant de plus en plus, au long de la période classique, comme une unité thématique forte.

${ }^{9}$ Ou leur « non-dépendance », plutôt.
} 
corpus, cependant proposant un renversement rhétorique plus fort que ses homologues. On peut le voir ci-dessous, en comparant l'exemple (10) aux exemples (11) et (12): tandis que Cependant marque une opposition majeure du propos, en inversant parfaitement les termes de l'argumentation ("les artisans doivent être fidèles, cependant il y a beaucoup d'artisans infidèles »), pourtant et neanmoins opèrent une adversation moins forte dans l'argument. En effet, ils n'inversent pas la valeur de vérité du premier élément argumentatif, mais apportent une précision ou un détail complémentaire qui, certes, s'opposent au précédent, mais dans une logique se rapprochant davantage de l'addition que de l'adversation. Il ne s'agit pas ici de contredire frontalement une thèse, mais d'anticiper l'opposition d'un contradicteur putatif en la devançant et en l'incluant dans sa démonstration. Sa valeur argumentative s'en retrouve alors amoindrie, ce qui permet de poursuivre le propos sans réorienter fondamentalement la programmation de l'information. Cela se voit notamment dans l'exemple (12) où Neanmoins pourrait se substituer sans mal par en effet ou effectivement sans compromettre la compréhension générale de l'extrait :

(10) Les devoirs des Artisans sont d'être fidéles dans leurs ouvrages, de n'être ni paresseux, ni débauchez; mais de s'attacher à leur vocation, sans pourtant négliger ce qui doit être toûjours la principale occupation des chrêtiens : Cependant combien d'artisans infidéles; combien de fénéants ; combien de débauchez ? Comment doivent se conduire les jeunes gens, de l'un \& de l'autre sexe ; \& quelles doivent être leurs œuvres. C'est de consacrer leurs beaux jours à Dieu [...] (Pictet, 1716).

(11) Il a porté nos pechez en son corps sur le bois, afin que mourans au peché, nous viuions à justice; Pourtant Saint iean dit que si nous cheminons en lumiere comme Dieu est lumiere, nous auons communion auec luy, \& le sang de son fils iesus-christ nous purge de tout peché, ne recognoissant le sang de iesus-christ estre alloüé qu'à ceux qui satisfốt à la fin \& au but de la foy $\&$ de l'euangile (Mestrezat, 1645).

(12) C'est donc par vne veritable inspiration du S. Esprit, que Siméon voyant éclater les premiers rayons de ce soleil naissant, s'écria dans son extase, Luc 2. lumiére pour l'éclaircissement des Nations! Neanmoins, c'est pour trois raisons considerables que Malachie, parlant icy au nom du seigneur, dit particulierement aus fideles iuifs, A vous, qui craignez mon Nom, se levera le Soleil de iustice [...] (Drelincourt, 1670).

Comme nous le savons, à la fin de la période classique, le signe du point a été réinvesti par les locuteurs surtout sous l'angle de "l'invention de la phrase ", en proposant une autre segmentation de l'unité infratextuelle fondamentale se concentrant sur une prédication principale et éliminant, partant, les "périodes à rallonge " qui démultipliaient leurs membres tout en gardant une unicité thématique patente (Siouffi, 2010 : 143). La phrase se faisait ainsi 
[...] l'assurance, et la visibilité première, d'une communication honnête respectant les étapes, et s'affranchissant du recours à l'ambiguïté tout autant que de l'éparpillement du sens dans une trame indistincte et sans limites (Siouffi, $1997: 238$ ).

Cependant, il n'en point de même du signe de la virgule. Celle-ci a, depuis longtemps, indiqué les membres incidents de l'énoncé : mais elle s'est également colorée d'une interprétation sémantique/argumentative spécifique, qui facilite la lecture en hiérarchisant l'importance du connecteur dans l'énoncé. Comme nous le disions plus haut, toute opération de segmentation est avant tout une opération de mise en lumière : isoler, par une virgule, un membre incident du point de vue syntaxique, c'est à l'inverse prendre le risque de lui conférer une importance supplémentaire du point de vue rhétorique. Il y a donc eu un double mouvement d'évolution contraire, l'un sur le plan de la forme, l'autre sur le plan du sens, qui rend difficile toute description positive de la ponctuation d'une part, des connecteurs de l'autre. Cela, du reste, est à mettre en perspective avec le genre des textes que nous avons analysés, ce paramètre ayant une incidence notable sur ces phénomènes. Nous renvoyons notamment aux études de Carine Skupien Dekens (2020 : 224-225), qui a noté dans un corpus de sermons religieux l'importance de la dimension oralisante dans ces pratiques de ponctuation : nous la rejoignons alors dans ces analyses. Nous renvoyons également à Goux (2016), pour une étude de l'influence de ce même paramètre dans l'emploi de certains outils de cohésion.

\subsection{Connecteur et dimensionnement informationnel}

Si nous nous concentrons cependant sur les connecteurs, peut-être l'élément le plus stabilisé de cette évolution dans notre corpus, nous aurions, et c'est ainsi que nous le résumerons ici, une évolution d'un statut de reposoir périodique à celui de membre de la structure phrastique. Ce dernier terme doit ici s'entendre indépendamment d'une analyse strictement syntaxique de l'énoncé, ou alors en la mâtinant d'une dimension sémantique ou rhétorique, argumentative, particulière, pas si éloignée, finalement, du sens que l'on donnait à ce terme à l'époque classique ( $c f$. Seguin, 1993). Il y a comme un mouvement inverse, lorsque nous mettons en lien le critère de la ponctuation et celui de la dynamique informationnelle de l'énoncé, au long de la période temporelle déterminée par notre corpus :

(i) Au début de l'époque classique, lorsque les connecteurs ont des rôles relativement « faibles " dans la dynamique périodique, à l'instar de pourtant et de néanmoins qui agissent davantage au sein des membres de la période et moins lors d'une articulation majeure de celle-ci comme le fait, dans notre corpus, cependant, puis ou ensuite, on tend à les encadrer par la ponctuation dont le rôle doit donc s'entendre comme une véritable aide à la lecture, plutôt qu'une aide à l'interprétation. Ils ont, à proprement parler et en reprenant la terminologie grammaticale du temps, un rôle de reposoir, 
marquant des endroits où la lecture " se repose ", s'arrête un instant dans le cadre d'une oralisation du propos par exemple, mais ces connecteurs ne permettent pas tant de construire le propos qu'à faciliter la compréhension générale de la période, à l'instar de la définition de reposoir que donne Richelet dans son dictionnaire :

Ce mot se dit en parlant de periodes Françoises. C'est un certain nombre de silabes dans chaque membre des belles périodes où l'oreille se repose agréablement. C'est environ le milieu de chaque membre. Ce mot de reposoir se dit aussi généralement de toutes les periodes $\&$ ce sont des pauses dans chaque periode $\&$ des endroits où l'oreille trouve quelque repos. [Toutes les periodes doivent avoir des reposoirs.] (Richelet, 1680 : II.302).

(ii) Plus l'on progresse au long du XVII ${ }^{\mathrm{e}}$ siècle cependant, plus cette tendance tend à s'inverser : ce sont, cette fois-ci, les éléments nécessaires à la compréhension du propos qui sont de mieux en mieux isolés par la ponctuation, tandis que les connecteurs moins importants pour la compréhension se fondent, sans démarcation graphique, dans la proposition ou dans la phrase qui les accueillent. Cette fois-ci, la ponctuation, l'encadrement par des virgules par exemple, est une aide déterminante pour la lecture : nous faisons notamment l'hypothèse que cette mise en lumière ainsi faite facilite l'analyse syntaxique de la phrase qui se constitue alors, en isolant les éléments qui ne dépendent pas directement de la valence verbale et participant, à sa façon, aux opérations de grammaticalisation qui s'observent. Il ne s'agit donc plus de reposoirs, mais bien de pivots argumentatifs déterminants qu'il convient, pour améliorer le confort du lecteur et s'assurer de sa compréhension, de mettre en lumière.

Toutes choses égales par ailleurs, nos analyses rejoignent celles de Carine Skupien Dekens (2020:215) qui évoquait, sur un corpus de sermons du temps, que c'était " [... la] combinaison des conjonctions (ou autres connecteurs) qui s'entendent, et de la ponctuation - qui ne s'entend que par une nuance intonative dépendant du prédicateur - qui marque à l'écrit la "ponctuation de l'oral" ".

Ces observations sont, de même, cohérentes avec les études s'intéressant à ces relations connecteurs/ponctuation en synchronie, à l'instar des études de Franck Neveu (2007 : 175) sur la langue contemporaine : "Ponctuer c'est déterminer un cadre linguistique à l'intérieur d'un espace textuel. Il s'agit donc d'une opération de dimensionnement des unités informationnelles, dans laquelle le placement d'un constituant en position d'ouverture ne saurait être tenu pour aléatoire ».

Nous apportons cependant une nuance décisive avec notre étude : la nécessité de distinguer dans ces problématiques la nature argumentative/rhétorique du connecteur considéré. S'il est vraisemblablement des effets généraux quant à cette problématique, notamment concernant l'identité des constituants en position initiale de séquence graphique (comme les études citées l'établissent sans ambiguïté aucune), le paramètre informationnel demeure crucial et a vraisemblablement influencé les pratiques 
de segmentation sur ces connecteurs précis, dans des proportions notables qu'il convient de prendre en compte.

\section{Conclusions}

Notre étude trace un parcours de la connexité à l'époque classique et de certaines de ses métamorphoses au sein de l'évolution cruciale de la période à la phrase. Moins qu'un mécanisme du changement, comme peuvent l'être les évolutions des constructions détachées (Combettes, 1998), des relais anaphoriques (Goux, 2017) ou des conjonctions de coordination (Goux, 2019a), ces connecteurs, et la thématique de la connexité en général, sont davantage des victimes de ce changement dans la mesure où ils en subissent directement les conséquences. Nous avons alors surtout constaté les concernant une reconfiguration des habitudes de production des énoncés, plutôt qu'un changement fondamental dans le système de la langue. Nous rappelons aussi que, de la même façon que l'emploi des connecteurs n'est pas une nécessité absolue pour la compréhension de l'énoncé, ceux-ci venant davantage faciliter la conduite du propos qu'ils n'aident proprement à le construire, la ponctuation les encadrant révèle davantage la façon dont les auteurs ou compositeurs considèrent les unités graphiques majeures de leur énoncé, qu'une volonté particulière de les régulariser.

En ce sens, et comme nous le notions en introduction, les paramètres du genre et de la séquence textuelle deviennent cruciaux dans cette perspective : des études récentes (à l'instar de Mounier, 2020) ont notamment montré que la programmation informationnelle influençait directement les pratiques de ponctuation, selon qu'il s'agissait d'assurer la continuité thématique et la liaison avec le contexte gauche, créer une rupture thématique ou d'agencer "syntactico-prosodiquement " un raisonnement (Mounier, 2020 : 205). Nous pouvons envisager qu'il en est de même pour l'emploi des connecteurs. Le choix que nous avons alors fait lors de la préparation de notre corpus, de nous concentrer sur la narration et l'argumentation d'une part, et de ne pas prendre en compte la dimension séquentielle des textes de l'autre, n'a pu que restreindre, de façon attendue, nos observations; mais en creux, ce choix n'a pu que mettre en lumière la nécessité de tenir compte de ce paramètre générique dans ce type d'études, malgré toutes ses difficultés d'application.

Nous désirons enfin, dans un ultime temps, confronter ces observations avec des tendances observables au sein de la presse contemporaine. En reprenant les mêmes connecteurs au sein du corpus de L'Est Républicain (2003), fourni par le CNRTL $(2005 \rightarrow)$ et en ne nous concentrant, cette fois-ci, qu'exclusivement sur le paramètre de la ponctuation, voici les résultats que nous obtenons : 


\begin{tabular}{|c|c|c|c|c|c|}
\cline { 2 - 6 } \multicolumn{1}{c|}{} & \multicolumn{2}{c|}{ Connecteurs additifs } & \multicolumn{3}{c|}{ Connecteurs adversatifs } \\
\hline Forme & Puis & Ensuite & Cependant & Néanmoins & Pourtant \\
\hline $\begin{array}{c}\text { Occurrences } \\
\text { Totales }\end{array}$ & 5037 & 2849 & 879 & 480 & 1598 \\
\hline $\begin{array}{c}\text { Emploi } \\
\text { de ponctuation }\end{array}$ & $\begin{array}{c}1997 \\
(39,6 \%)\end{array}$ & $\begin{array}{c}147 \\
(5,2 \%)\end{array}$ & $\begin{array}{c}187 \\
(21,9 \%)\end{array}$ & $\begin{array}{c}29 \\
(6 \%)\end{array}$ & $\begin{array}{c}187 \\
(11,7 \%)\end{array}$ \\
\hline
\end{tabular}

Tableau 4. Ponctuation et connecteurs dans L'Est Républicain (2003).

Si nous comparons ces résultats avec ceux relevés précédemment, la tendance observée semble se confirmer : tandis qu'un connecteur additif comme ensuite n'est pas particulièrement mis en lumière par la ponctuation, puis l'est bien davantage. Parmi les adversatifs, même constat : cependant est plus isolé typographiquement dans la phrase que pourtant et néanmoins, qui se moulent davantage dans la logique phrastique et sont alors davantage interprétés comme des connecteurs argumentatifs à l'importance moindre dans la compréhension générale de l'énoncé et donc non mis en lumière par la ponctuation, et ce malgré leur statut d'adverbe de liaison ne relevant ni de la valence verbale, ni d'un syntagme en particulier dans la phrase.

Aussi, et même si l'on ne saurait véritablement offrir une histoire uniforme de la ponctuation tant celle-ci dépend tant des tendances stylistiques propres à chaque auteur, des pratiques éditoriales, des dynamiques de genres et de séquences textuelles et de la programmation de l'information, les tendances relevées par l'intermédiaire de notre étude sur corpus dessinent un parcours spécifique où se mêlent sémantique, rhétorique et grammaire de texte. À partir de ces premiers résultats, deux grands chantiers sont alors à entreprendre pour approfondir notre connaissance de la connexité en diachronie.

Tout d'abord, il convient de mener une étude du même ordre sur les marqueurs disjonctifs du type d'une part... d'autre part, en association peut-être des conjonctions et locutions conjonctives ou et soit (que). Cette problématique étant liée à la question de l'évolution et de la stabilisation des systèmes corrélatifs en diachronie, elle est d'autant plus pertinente pour l'étude de l'évolution de la dépendance syntaxique de l'ancien français au français moderne et pour l'étude de la façon dont les unités infratextuelles étaient pressenties par les auteurs du temps.

Dans un second temps, il serait sans doute pertinent de réinvestir nos observations dans une étude plus large, portant sur la façon dont l'argumentation et la démonstration, tant scientifique que littéraire, était conduite et considérée dans le temps à l'instar des travaux de Callemein \& Goux (2020) sur le texte juridique. La programmation de l'information, l'emploi des connecteurs et de la ponctuation s'inscrivent certes dans un cadre générique et séquentiel, dans le sens textuel du terme, mais ils dépendent également du sous-genre littéraire - au sens large du terme - dans lequel nous l'envisageons. 
Un récent numéro de Langue française (Basso Fossali, 2020) montre ainsi que dans le domaine pourtant étroit des "discours d'incitation de l'action ", dits encore "discours programmateurs", les différences étaient grandes entre une recette de cuisine, une notice de jeu ou le mode d'emploi d'un médicament : on peut donc envisager qu'il en est de même, pour l'emploi des connecteurs et de la ponctuation, entre une argumentation conduite dans le cadre d'un essai philosophique, d'un texte narratif fictionnel ou d'un traité médical. Les grands corpus numérisés nous permettront sans doute, dans les années venantes, de mettre au jour ces différentes variables et d'affiner ce faisant les contours des évolutions décisives qu’ont connu les énoncés en français préclassique et classique.

\section{RÉFÉRENCES BIBLIOGRAPHIQUES}

ADAM, Jean-Michel (1987) : "Textualité et séquentialité. L'exemple de la description». Langue française, 74, 51-72.

ADAM, Jean-Michel \& Michel FAYOL (1989) : «Présentation ». Langue française, 81, 3-4.

APOTHÉLOZ, Denis (1995) : Rôle et fonctionnement de l'anaphore dans la dynamique textuelle. Paris, Droz.

AYRES-BENNETT, Wendy \& Philippe CARON (2016) : «Periodization, translation, prescription and the emergence of Classical French ». Transactions of the Philological Society, $114: 3,339-390$.

BASSO FOSSALI, Pierluigi [éd.] (2020) : Langue française, 206 [Incitation à l'action et genres de discours programmateurs].

BERRENDONNER, Alain \& Marie-José REICHLER-BÉGUELIN (1989) : «Décalages : les niveaux de l'analyse linguistique ». Langue française, 81, 99-125.

BERRENDONNER, Alain et al. (2012) : Grammaire de la période. Bern, Peter Lang.

BRASSART, Dominique-Guy (1993) : "Effet des connecteurs sur le rappel des textes par des enfants de 8 et 10 ans bons et mauvais lecteurs et des adultes ". L'Année psychologique, $93: 4,507-525$.

CALlemein, Gwenaëlle \& Mathieu GOUX (2020) : «Les “questions de droit” dans la Coutume réformée de Henri Basnage (1678) : analyse d'un dispositif explicatif. "L'information grammaticale, 120, 31-38.

Charolles, Michel \& Denis Vigier, (2005) : «Les adverbiaux en position préverbale : portée cadrative et organisation des discours ». Langue française, 148, 9-30.

COMBETTES, Bernard (1983) : Pour une grammaire textuelle. Bruxelles, De Boeck.

COMBETTES, Bernard (1994) : «Une approche diachronique des connecteurs et des modalisateurs ». Pratiques, 84, 55-67.

COMBeTTES, Bernard (1998) : Les Constructions détachées en français. Paris, Ophrys. 
COMBETTES, Bernard (2003) : "Présentation », in Bernard Combettes (éd.), Évolution et variation en français préclassique. Études de syntaxe. Paris, Honoré Champion, 3-18.

DAUVOIS, Nathalie \& Jacques DÜRRENMATT (2011) : La Ponctuation à la Renaissance. Paris, Classiques Garnier.

DEMONET, Marie-Luce (2011) : " Ponctuation spontanée et ponctuation civile », in Nathalie Dauvois et Jacques Dürrenmatt (éd.), La Ponctuation à la Renaissance. Paris, Classiques Garnier, 129-148.

FOURNIER, Nathalie (1998) : "Quelques problèmes concernant l'anaphore et les marqueurs anaphoriques dans Nicomède ». L'Information grammaticale, 76, 25-28.

FOURNIER, Nathalie (2002). Grammaire du français classique. Paris, Belin.

Goux, Mathieu (2016) : « Du fait linguistique au(x) genre(s) : oralité et scripturalité à l'époque classique ». Essais, 7, 137-151.

GOUX, Mathieu (2017) : "De la période à la phrase : le témoin lequel», in Sophie Prévost et Benjamin Fagard (éds), Le français en diachronie : dépendances syntaxiques, morphosyntaxe verbale, grammaticalisation. Paris, Peter Lang, 133-154.

GOUX, Mathieu (2019a) : "Les subordonnées relatives coordonnées en français classique: entre référence et prédication ", in Daniéla Capin, Julie Glikman, Vanessa Obry \& Thierry Revol (éds), Le français en diachronie : moyen français, segmentation des énoncés, linguistique textuelle. Strasbourg, EliPhi, 107-120.

GOUX, Mathieu (2019b) : Le Pronom-déterminant relatif LEQUEL en français préclassique et classique (1580-1720). Paris, Garnier.

GouX, Mathieu (2020) : «Commencer en continuant : du rôle des constructions détachées en -ant en tête de chapitre. L'exemple d'Oudin, de Rosset et de Vaugelas ". Scolia. Revue de Linguistique, 34 [Peter Helland, ed., Thématisation et périphéries de la phrase], 115-131. DOI : https://doi.org/10.4000/scolia.1362.

GRÉVISSE, Maurice \& André GoOSSE (2016) : Le Bon Usage. Louvain-la-Neuve, De Boeck Supérieur.

LE GOFFIC, Pierre (2011) : «Phrase et intégration textuelle ». Langue française, 170, 11-28.

MOUNIER, Pascale (2020) : «L'agencement thème-rhème à l'échelle de l'énoncé dans le roman vers 1530 ». Verbum, XLI : 2, 185-208.

NeveU, Franck (2007) : «Détachement et connexité ». in Claude Guimier (dir.), Eléments de relation : de la phrase au texte. Caen, Presses Universitaires de Caen, 165-177.

PRÉVOST, Sophie (2001) : La postposition du sujet en français aux XVet $X V I^{e}$ siècles. Paris, CNRS éditions.

RAULT, Julien (2020) : "L'achèvement de la phrase au XVIII siècle : point et contre-point ". Verbum, XLI : 2, 323-336.

RÉGNIER-DeSMARAIS, ABBÉ (1706) : Grammaire françoise. Paris, Jean-Baptiste Coignard.

RICHELET, César-Pierre (1680) : Dictionnaire françois. Genève, Jean Herman Widerhold. URL : https:/gallica.bnf.fr/ark:/12148/bpt6k509323.image 
RIEGEL, Martin et al. (2014) : Grammaire méthodique du Français. Édition Revue et Augmentée. $5^{\mathrm{e}}$ édition. Paris, Presses Universitaires de France.

ROSSARI, Corinne (1998) : "Analyse contrastive, grammaticalisation et sémantique des connecteurs ». Travaux de Linguistique, 36, 115-126.

ROSSARI, Corinne (2002) : «Les adverbes connecteurs : vers une identification de la classe et des sous-classes ". Cahiers de linguistique française, 24, 11-43.

SEGUIN, Jean-Pierre (1993) : L'Invention de la phrase au XVIII' siècle : contribution à l'histoire du sentiment linguistique français. Louvain, Peeters.

SIOUfFI, Gilles (1997) : "Quelques remarques sur la phrase au XVII ${ }^{e}$ siècle ", in Catherine Rannoux \& Jacques Dürrenmatt (dir.), La Phrase. Mélanges offerts à Jean-Pierre Seguin. Poitiers, La Licorne, 233-243.

SIOUfFI, Gilles (2010) : Le Génie de la langue française. Études sur les structures imaginaires de la description linguistique à l'Âge classique. Paris, Honoré Champion.

SIOUfFI, Gilles [coord.] (2020) : Phrase et période entre les XVI et XVIII' siècles. Verbum, XLI : 2.

SKUPIEN DEKENS, Carine (2020) : «Ponctuation et cohésion : ce que les premiers mots nous disent. Étude sur un corpus de sermons protestants du XVI ${ }^{\mathrm{e}}$ au XVIII ${ }^{\mathrm{e}}$ siècle ». Verbum, 41: 2, 209-227.

\section{ANNEXE}

\section{Sources primaires et corpus}

BerNiER, François (1684) : Abrégé de la philosophie de Gassendi. Paris, Anisson, Posuel et Rigaud. Cote Frantext: Q535 à Q539, Q543 \& Q545.

BÉZÉ, Théodore de (1593) : "Sermon deuxième». Sermon sur l'histoire de la resurrection de nostre seigneur Iesus Christ. Paris, Jean Le Preux. Cote Frantext : E199.

BOISGUILBERT, Pierre de (1695) : Le détail de la France sous le règne présent. Paris, G. De Backer. Cote Frantext: Q987 \& Q988.

CHIFLET, Laurent (1659) : Essay d'une parfaite grammaire de la langue françoise. Anvers, Jacques Van Meurs. Reprod. (1973). Genève, Slatkine Reprints.

Colombat, Bernard, Jean-Marie Fournier \& Wendy Ayres-Bennet [éds] (2011) : Grand Corpus des Grammaires Françaises, des Remarques et des Traités sur la Langue (XIVE$X V I I^{e}$ s.). Paris, Classiques Garnier et al (2011). URL : https://www.classiques-garnier.com/numerique

DRELINCOURT, Laurent (1670) : Le salutaire lever du soleil de justice, ou, Sermon sur Malachie, chap. IV, vers. 2. Paris, Jean Antoine et Samuel de Tournes. Cote Frantext : E208.

Frantext $=$ Montémont, Véronique [dir.] $(2002 \rightarrow):$ Frantext. Outil de consultation de ressources informatisées sur la langue française. Nancy, ATILF. URL : http://www.frantext.fr 
HaRlay DE SANCY, Nicolas de (1610). Discours sur l'occurrence de ses affaires. Paris, Champion. Cote Frantext : S000.

L'Est Républicain (2003), in CNRTL, Centre National de Ressources Textuelles et Lexicales, (2005 $\rightarrow$, en ligne), CNRS-ATILF. URL : https://www.cnrtl.fr/accueil/contributeurs.php

LA Forge, Louis de (1661) : Traitté de l'esprit de l'homme... Paris, Bobin et Le Gras. Cote Frantext: E247.

LE FAUCHEUR, Michel (1632) : Sermon des souffrances des fidelles \& de leur gloire. Paris, Michel et Pierre Auvray. Cote Frantext: E204.

MARINELLO, Giovanni (1609) : Les maladies des femmes \& remedes d'ycelles en trois livres. Paris, J. Berjot. Cote Frantext: V000.

Mestrezat, Jean (1645) : Sermon, sur le pseaume CXXX. Vers. 1. 2. 3. \& 4. Paris, Samuel Petit. Cote Frantext : E205.

PICTET, Bénédict (1716) : L'examen des chrétiens, ou Sermon sur Apoc. III. vs. 1. 2. 3. Paris, Jean Antoine Querel. Cote Frantext: E216.

Rossal, Pierre (1701) : Les droits de Dieu, \& ceux des souverains, ou Sermon sur le couronnement de sa Majesté, Frédéric roi de Prusse. Paris, Jean-David Bergmann. Cote Frantext : E215. 